\title{
Electromagnetic scattering beyond the weak regime: Solving the problem of divergent Born perturbation series by Padé approximants
}

\author{
T. A. van der Sijs, ${ }^{1}$ O. El Gawhary, ${ }^{1,2,{ }^{*}}$ and H. P. Urbach ${ }^{1}$ \\ ${ }^{1}$ Optics Research Group, Imaging Physics Department, Delft University of Technology, Van der Waalsweg 8, 2628 CH Delft, Netherlands \\ ${ }^{2}$ VSL Dutch Metrology Institute, Thijsseweg 11, 2629 JA Delft, Netherlands
}

(Received 19 November 2019; accepted 31 January 2020; published 13 March 2020)

\begin{abstract}
Electromagnetic scattering is the main phenomenon behind all optical measurement methods where one aims to retrieve the shape or physical properties of an unknown object by measuring how it scatters an incident optical field. Such an inverse problem is often approached by solving, several times, the corresponding direct scattering problem and trying to find the best estimate of the object which is compatible with a set of measurements. Despite the existence of numerical methods, a powerful way to solve those direct problems would be to use a perturbation approach where the field is expressed as a series, known as the Born series. The advantage of a perturbation approach stems from the fact that each term of the series has a clear physical meaning and can unveil much more about the scattering process than a purely numerical approach can offer. This method is however unpractical under so-called strong-scattering conditions because the corresponding Born series strongly diverges. In this work, we will show how to solve this problem by employing Padé approximants and how to treat electromagnetic problems well beyond the weak-scattering regime. This approach can represent an important building block to the application of the Born series to direct and inverse problems, with potential applications in superresolution, optical metrology, and phase retrieval.
\end{abstract}

DOI: 10.1103/PhysRevResearch.2.013308

\section{INTRODUCTION}

Contactless measurement techniques based on light are of growing relevance due to their noninvasiveness, speed, and integrability in industrial processes. In very general terms, the main idea behind these methods is that when a known electromagnetic field, typically in the visible range, interacts with an unknown object, information about the object gets encoded in the scattered field produced by such interaction. Applications often require that the scattered field (or its intensity only) is measured at distances of many wavelengths from the object, a condition usually referred to as the far-field regime. By measuring this scattered field, and knowing the laws of physics that govern the interaction, useful information about the object can be retrieved. The determination of the object from the measured scattered field requires solving a nonlinear inverse problem, which can be formally formulated by means of the Lippmann-Schwinger equation. The nonlinear nature of the problem stems from the fact that the scattered field inside the object is unknown and depends strongly on the object itself (namely, its geometry and/or its permittivity) [1]. Inverse electromagnetic problems are central in modern technologies, of which the most important example probably is metrology in

\footnotetext{
*o.elgawhary@tudelft.nl

Published by the American Physical Society under the terms of the Creative Commons Attribution 4.0 International license. Further distribution of this work must maintain attribution to the author(s) and the published article's title, journal citation, and DOI.
}

the semiconductor sector. Those problems are usually difficult to solve and in many cases methods to approach them rely on iteratively solving the direct problem starting from some prior knowledge of the object. At every step, the object guess is updated and the scattered electromagnetic field is updated by solving the direct scattering problem for the updated estimate of the object, until the iteration converges. The advantage of this approach is that, contrarily to inverse problems, direct problems are mathematically well-posed and there exist many different numerical tools-finite element methods, finite difference time domain, rigorous coupled wave analysis, just to name a few-to solve them nowadays, even for very complex geometries [2-5]. Resorting to those numerical solvers has, however, the downside of losing a comprehensible connection with the physical mechanisms that lead to the solution. Thus, in view of their applications to solving inverse problems, an analytical approach to direct scattering problems is still highly valuable. A way to achieve that would be to try to solve the Lippmann-Schwinger equation for the scattered field using a perturbative approach. In this method, the field is expressed as a sum of terms which represent the different perturbation orders. When solving for the total field, the zeroth order represents the unperturbed solution, while the first order is denoted as the Born approximation [6-10]. At every step a new term of the series is computed and added to the sum, which leads to a possibly more accurate estimate of the actual solution. As we will recall in Sec. II, breaking down the solution into different contributions allows for keeping the connection with the physics of the problem as each term of the series has a clear physical meaning, which is a clear advantage of perturbation series over numerical solvers. Like 
all perturbation methods, when the scattering is weak, the first order solution (the Born approximation) provides already a good estimate of the solution and all higher order terms of the series can be safely neglected. On the other hand, when higher-order terms are large, the scattering is said to be strong and one must go beyond the Born approximation. The Born series is just one example of perturbation series which are encountered in different areas of physics. Applications of perturbation series are presented in standard books on quantum mechanics and classical and quantum electrodynamics as a way to solve algeabric, integral or differential equations for which a close-form solution is not available. In the case of the Born series, most standard books do not stress enough that when the interaction between the field and the object is strong, in a sense which will be clarified later on, the Born series diverges, which frustrates the benefit of the whole approach [11]. This problem has been originally encountered in particle physics, where different methods have been investigated to circumvent it, ranging from iterative methods [12], to transforming the original Lippmann-Schwinger equation from a Fredholm to a Volterra structure by renormalization [13], using other transformation-based methods [14] or through nonlinear resummation techniques $[15,16]$. More specifically, in Ref. [15], Tani has theoretically investigated how Padé approximants are able to reproduce several terms of an original Born series exactly up to the $2 N$-th order, where $N$ is the order of the approximant, but with a well-defined radius of convergence larger than that of the original Born series. Padé approximants have also been applied to scattering series in three-body problems and elastic particle scattering [17,18]. In this work we will consider one-dimensional (1D) and twodimensional (2D) scalar scattering problems in electromagnetism that lie far beyond the weak-scattering regime. These cases are usually deemed unapproachable using a perturbative approach as they typically lead to exponentially diverging series. Our goal is to show that one can still make use of the information provided by the terms of the divergent Born series and obtain accurate results out of perturbative methods, even in the strong-scattering regime. As explained above, having a tool available to solve forward scattering problems through a semianalytical approach is extremely useful in the area of inverse problems and our interest is to develop a tool that will allow to integrate multiple-scattering effects into optical metrology techniques [19-24]. In Sec. II, we start by laying out the general scalar scattering problem and its perturbative solution. We also introduce Padé approximants and how they can be applied to this problem. In Sec. III, we elaborate on this approach, by applying it to the 1D case of a slab placed in a vacuum, after which we extend the procedure to the $2 \mathrm{D}$ case of an infinitely long cylinder in Sec. IV. Both cases will allow us to perform some rigorous quantitative analysis to define the boundary between the weak- and strong-scattering regimes. We conclude the paper in Sec. V and discuss the implications of this new approach.

\section{RESUMMATION OF THE BORN SERIES WITH PADÉ APPROXIMANTS}

We start by considering one of the scalar components of the monochromatic electric field in a region of space where an object of relative electric permittivity $\varepsilon_{r}(\mathbf{r})$ is present. The permittivity may be a complex quantity as in the case of an absorbing medium. We will consider monochromatic fields which depend on time by the factor $\exp (-i \omega t)$ where $\omega>0$. This factor is omitted from all formulas. The governing equation for the field is the Helmholtz equation and for simplicity we apply the scalar approximation and consider the scalar version of the Helmholtz equation, which reads

$$
\nabla^{2} U(\mathbf{r})+k_{0}^{2} \varepsilon_{r}(\mathbf{r}) U(\mathbf{r})=0,
$$

where $k_{0}=2 \pi / \lambda, \lambda$ is the wavelength of the monochromatic field in vacuum, and $\mathbf{r}=(x, y, z)$. We can rewrite this equation in the following way:

$$
\nabla^{2} U(\mathbf{r})+k_{0}^{2} U(\mathbf{r})=-k_{0}^{2} \Delta \varepsilon_{r}(\mathbf{r}) U(\mathbf{r}),
$$

where $\Delta \varepsilon_{r}(\mathbf{r})=\varepsilon_{r}(\mathbf{r})-1$ is the permittivity contrast, namely the difference in relative permittivity between the object and the environment (which is supposed to be vacuum/air in this case). Equation (2) can be written in an integral form

$$
U(\mathbf{r})=U_{i}(\mathbf{r})+k_{0}^{2} \int_{\mathbf{r}^{\prime}} G\left(\mathbf{r}, \mathbf{r}^{\prime}\right) U\left(\mathbf{r}^{\prime}\right) \Delta \varepsilon_{r}\left(\mathbf{r}^{\prime}\right) d \mathbf{r}^{\prime},
$$

with $U_{i}(\mathbf{r})$ the known incident field and $G\left(\mathbf{r}, \mathbf{r}^{\prime}\right)$ the free-space Green's function satisfying $\left[\nabla^{2}+k_{0}^{2}\right] G\left(\mathbf{r}, \mathbf{r}^{\prime}\right)=-\delta\left(\mathbf{r}-\mathbf{r}^{\prime}\right)$. We can rewrite Eq. (3) in terms of the scattered field $U_{s}(\mathbf{r})=$ $U(\mathbf{r})-U_{i}(\mathbf{r})$ as

$$
U_{s}(\mathbf{r})=k_{0}^{2} \int_{\mathbf{r}^{\prime}} G\left(\mathbf{r}, \mathbf{r}^{\prime}\right)\left[U_{i}\left(\mathbf{r}^{\prime}\right)+U_{s}\left(\mathbf{r}^{\prime}\right)\right] \Delta \varepsilon_{r}\left(\mathbf{r}^{\prime}\right) d \mathbf{r}^{\prime} .
$$

Equation (3), known under the name of Lippmann-Schwinger equation, is an integral equation for the unknown field $U(\mathbf{r})$. Two very different types of problems require to solve Eq. (3). The first one is when the incident field $U_{i}(\mathbf{r})$ and permittivity contrast $\Delta \varepsilon_{r}(\mathbf{r})$ are both known and the total field $U(\mathbf{r})$ has to be computed. This is called the forward scattering problem. In that case, Eq. (3) is a linear integral equation for the unknown $U(\mathbf{r})$. If, on the other hand, the incident field $U_{i}(\mathbf{r})$ is known and the scattered field $U(\mathbf{r})$ far from the object is measured, and one aims to determine the properties of the object, namely $\Delta \varepsilon_{r}(\mathbf{r})$, then the problem is an inverse scattering problem which is nonlinear because the integral in Eq. (4) depends on the unknown contrast $\Delta \varepsilon_{r}(\mathbf{r})$ directly as well as through the unknown scattered field $U_{s}(\mathbf{r})$. In both cases, it can be very useful to resort to a perturbative approach to find the expression of the scattered field $U_{s}(\mathbf{r})$ inside the scatterer. Like all perturbation methods, a generic parameter, say $\sigma$, is introduced in the original equation, which becomes

$$
\nabla^{2} U^{(\sigma)}(\mathbf{r})+k_{0}^{2} U^{(\sigma)}(\mathbf{r})=-k_{0}^{2} \sigma \Delta \varepsilon_{r}(\mathbf{r}) U^{(\sigma)}(\mathbf{r}) .
$$

The total field $U^{(\sigma)}(\mathbf{r})$ is then written as the Born series:

$$
U^{(\sigma)}(\mathbf{r})=\sum_{\ell=0}^{\infty} U_{\ell}(\mathbf{r}) \sigma^{\ell},
$$

where $U_{\ell}(\mathbf{r})$ are the different perturbation orders. The solution to the original problem is obtained by setting $\sigma=1$. The key idea behind perturbation methods is that one replaces a problem which is difficult to solve [namely, Eq. (2)] by a sequence of problems, each of which is easier to solve than 
the original problem. By substituting Eq. (6) into Eq. (3), in which $\Delta \varepsilon_{r}(\mathbf{r})$ is replaced by $\sigma \Delta \varepsilon_{r}(\mathbf{r})$, we get

$$
\begin{aligned}
\sum_{\ell=0}^{\infty} U_{\ell}(\mathbf{r}) \sigma^{\ell}= & U_{i}(\mathbf{r})+\sigma k_{0}^{2} \int_{\mathbf{r}^{\prime}} \Delta \varepsilon_{r}\left(\mathbf{r}^{\prime}\right) G\left(\mathbf{r}, \mathbf{r}^{\prime}\right) \\
& \times \sum_{\ell=0}^{\infty} U_{\ell}\left(\mathbf{r}^{\prime}\right) \sigma^{\ell} d \mathbf{r}^{\prime} .
\end{aligned}
$$

Equating the same powers in $\sigma$ in Eq. (7) gives, for the first few terms of the expansion in Eq. (6),

$$
\begin{aligned}
& U_{0}(\mathbf{r})=U_{i}(\mathbf{r}), \\
& U_{1}(\mathbf{r})=k_{0}^{2} \int_{\mathbf{r}^{\prime}} \Delta \varepsilon_{r}\left(\mathbf{r}^{\prime}\right) G\left(\mathbf{r}, \mathbf{r}^{\prime}\right) U_{0}\left(\mathbf{r}^{\prime}\right) d \mathbf{r}^{\prime}, \\
& U_{2}(\mathbf{r})=k_{0}^{2} \int_{\mathbf{r}^{\prime}} \Delta \varepsilon_{r}\left(\mathbf{r}^{\prime}\right) G\left(\mathbf{r}, \mathbf{r}^{\prime}\right) U_{1}\left(\mathbf{r}^{\prime}\right) d \mathbf{r}^{\prime},
\end{aligned}
$$

and so on. When terms of the series of order higher than $U_{1}(\mathbf{r})$ are considered, multiple-scattering effects are taken into account. The first order of Eq. (8) is obtained by assuming that the excited secondary source density inside the object is entirely determined by the incident field. The second- and higher-order terms are obtained by taking into account that the source density at a certain point in the object is modified by the fields radiated by other secondary sources in the object. Although the perturbative approach to solve problems such as Eq. (2) seems feasible, its application to actual problems turns out to be rarely possible, except for all those cases where the optical contrast is low and the scatterer is weak as, for instance, in the $\mathrm{x}$-ray range of the electromagnetic spectrum. In fact, as soon as the scatterer cannot be considered weak, in a sense that will be quantified below, the Born series diverges for $\sigma=1$ and the expression in Eq. (6) can not be used to compute the field directly. The goal of this article is to show how to recover an accurate solution of Eq. (1) by using the perturbative expansion even in the case of strong scatterers. In order to find a solution to the divergence problem, in this work, we will replace the divergent Born series by a sequence of Padé approximants derived from the available terms of the Born series. More specifically, in the Padé method a sequence of rational functions of the type

$$
P_{M}^{N}(\sigma, \mathbf{r})=\frac{\sum_{n=0}^{N} A_{n}(\mathbf{r}) \sigma^{n}}{\sum_{m=0}^{M} B_{m}(\mathbf{r}) \sigma^{m}}
$$

is generated, where $B_{0}(\mathbf{r})=1$. In Eq. (9), $P_{M}^{N}(\sigma)$ is the Padé approximant of order $M, N$. Among the different sequences of rational functions one can generate, we will, in this work, focus only on the symmetric case where $M=N$, simply referring to the function as a Padé rational of order $N$. The coefficients $A_{n}(\mathbf{r})$ and $B_{n}(\mathbf{r})$ in Eq. (9) are determined by equating Eq. (9) for the given order $N$ to Eq. (6) where the Born series is truncated after $2 N+1$ terms. This allows these coefficients to be expressed as functions of the perturbation orders $U_{\ell}(\mathbf{r}), \ell=0, \ldots, 2 N$. The expressions of $A_{n}(\mathbf{r})$ and $B_{n}(\mathbf{r})$ for some $N$ in terms of the orders $U_{0}(\mathbf{r}), \ldots, U_{2 N}(\mathbf{r})$, are given in Appendix A. The approximant can be calculated for all points inside and outside the scatterer directly. Alternatively, once the approximant inside the object is found, it is possible to calculate $U(\mathbf{r})$ outside using Eq. (3). Considering computational efficiency, it suffices to solve a rather small system of equations (for the coefficients of the numerator and denominator) in every point of the scatterer, contrary to numerical solvers. Hence, very low amounts of memory are required compared to numerical methods. The powerful feature of this approach lies in the fact that the sequence $P_{0}^{0}, P_{1}^{1}, P_{2}^{2}, \ldots, P_{N}^{N}$ (evaluated at $\sigma=1$ ) can converge to the actual solution of the problem even though the original perturbation series does not. The reason that the Born series diverges for $\sigma=1$ in the case of medium and high contrast, is that the solution $U^{(\sigma)}(\mathbf{r})$ has, as a function of $\sigma$, at least one pole in the complex plane with a modulus smaller than 1 . In such a case the Born series, which is the power series of $U^{(\sigma)}(\mathbf{r})$ around $\sigma=0$, has a radius of convergence smaller than 1 and hence the Born series diverges for $\sigma=1$. The Padé approximant is a rational function which can represent poles and which therefore can be used to find accurate approximations for fields where the power series diverges. By equating both (for $\sigma=1$ ), one can derive the coefficients of the Padé approximant and generate a sequence of rational functions which can converge although the original power series does not. Equating the power series and the Padé approximant implies that one assumes that both approximations have a region of convergence in the complex plane in common. Interestingly, this means that the terms of the original Born series contain all information about the scattered field, despite the fact that the series diverges. In a sense, the Born series is analytically continued by re-representing the series into a sequence of Padé approximants that converges in a larger domain in the complex plane. Rigorous mathematical proof on the universal convergence of Padé approximants is limited to the special case of Stieltjes functions [11], which is difficult to check and seems unlikely to be the case.

\section{STRONG-SCATTERING REGIME OF A SCALAR FIELD BY A ONE-DIMENSIONAL OBJECT}

Consider an incident field $U_{i}(z)=\exp \left[i k_{0} z\right]$ which impinges on an object, consisting of a medium of permittivity $\varepsilon_{0} \varepsilon_{r}(z)$ and thickness $d$, immersed in a medium of permittivity $\varepsilon_{0}$. We remind that $\varepsilon_{r}$ denotes the relative permittivity of the object and $\varepsilon_{0}$ is the permittivity of vacuum. See Fig. 1 .

We have chosen this specific example because it is possible to obtain a closed-form expression of the total field $U(z)=$ $U_{i}(z)+U_{s}(z)$ by just solving the 1D Helmholtz equation $d^{2} U(z) / d z^{2}+k_{0}^{2} \varepsilon_{r}(z) U(z)=0$ in the three domains $z<z_{1}$, $z_{1} \leqslant z \leqslant z_{1}+d$ and $z>z_{1}+d$ and connecting the solutions by imposing boundary conditions for the field at the two interfaces. In Appendix B, we provide the complete expression of the analytical solution. In Fig. 1, we show the absolute value of the total field for three different cases, where $\Delta \varepsilon_{r}=$ 4, $\Delta \varepsilon_{r}=4+i$ and $\Delta \varepsilon_{r}=4+4 i$, and with parameters $\lambda=$ $800 \mathrm{~nm}$ and $d=500 \mathrm{~nm}$. These values of $\Delta \varepsilon_{r}$ are chosen for two reasons. First of all, they all correspond to scattering problems in the strong-scattering regime, leading to a divergent Born series, while representing the different cases of a pure dielectric and of absorptive media of different absorption levels. Secondly, those values have been used in some previous work by different authors and the reader can use 
(a)
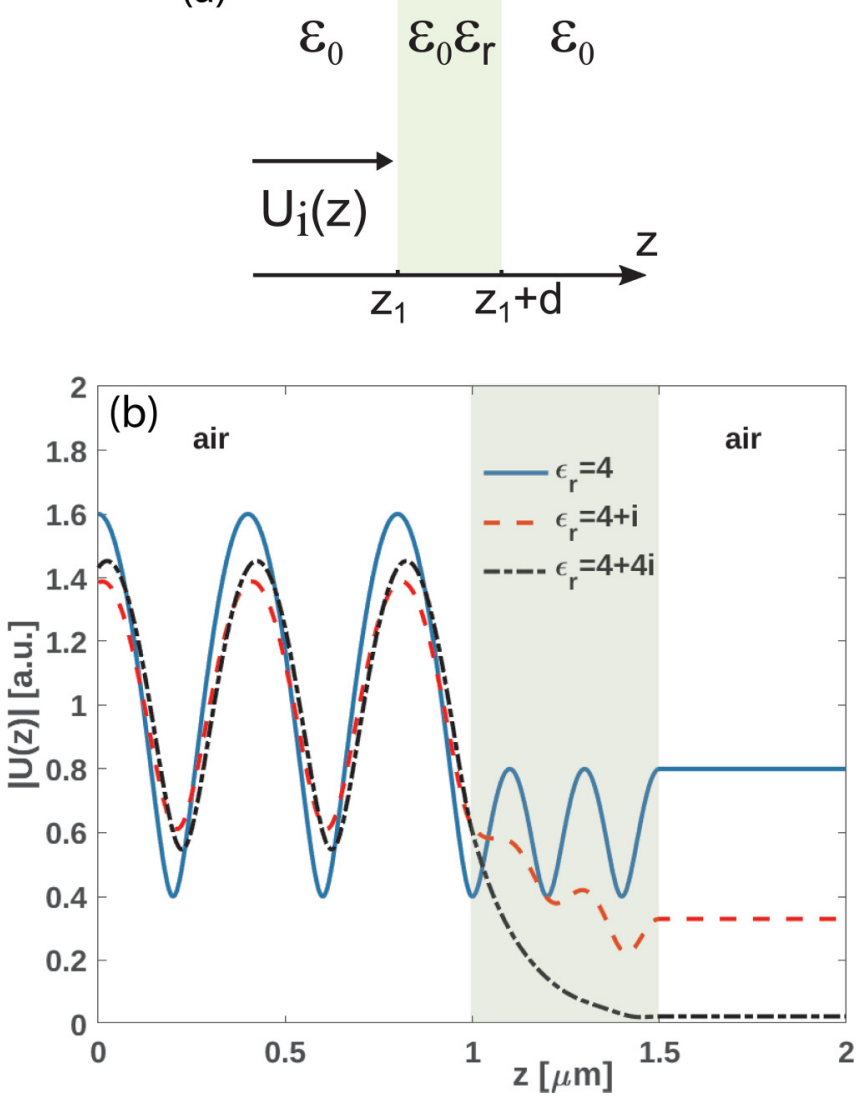

FIG. 1. (a) Geometry of the 1D electromagnetic problem studied. The scatterer has relative permittivity $\varepsilon_{r}$ and thickness $d$. (b) Absolute value of the analytic solution of the total field $U(z)$. Each curve corresponds to a different medium: $\varepsilon_{r}=4$, blue-solid line, $\varepsilon_{r}=4+i$, red-dashed line, $\varepsilon_{r}=4+4 i$ black-dot dashed line. Also, $z_{1}=1 \mu \mathrm{m}$ and $d=500 \mathrm{~nm}$.

those works, as well as other independent methods available, as an independent reference for comparison [25,26]. Because analytical solutions are rarely available, it is important to find alternative ways to solve the scattering problem which can be broadly applied. One method is to resort to the perturbation series approach as indicated in Eq. (6) and making use of a known analytical solution is a good way to assess the success of perturbative methods. While all terms of the series can be computed by numerical integration, in this work we have also computed the first few terms of the series analytically. This is useful because one can validate in this way the numerical integrations used to compute the terms of the series. Moreover, the analytical expressions help to identify the transition between the weak- and strong-scattering regime and the physical parameters that control that transition.

More specifically, we have computed the analytical expressions of the first three terms of the Born series, $U_{0}(z), U_{1}(z), U_{2}(z)$, for the case where the incident field is a plane wave $U_{i}(z)=\exp \left[i k_{0} z\right]$. The terms are calculated by means of Eq. (8), in which we use the 1D free-space Green's function $G\left(z, z^{\prime}\right)=i \exp \left[i k_{0}\left|z-z^{\prime}\right|\right] /\left(2 k_{0}\right)$. The reader interested in all details can refer to Appendix $C$, where those analytical expressions are explicitly given. Although the
TABLE I. Absolute value of perturbation orders, from 0th to 14th order, for $\varepsilon_{r}=4, \lambda=800 \mathrm{~nm}$ at position $z=1.5 \mu \mathrm{m}$. The correct value of the absolute value of the total field is $|U(z=1.5 \mu \mathrm{m})|=$ 0.80 .

\begin{tabular}{lc}
\hline \hline Perturb. order & Absolute value \\
\hline 0 & 1.0 \\
1 & 5.9 \\
2 & $1.8 \times 10^{1}$ \\
3 & $3.7 \times 10^{1}$ \\
4 & $4.3 \times 10^{1}$ \\
5 & $4.3 \times 10^{2}$ \\
6 & $2.4 \times 10^{3}$ \\
8 & $2.4 \times 10^{4}$ \\
10 & $1.3 \times 10^{5}$ \\
12 & $4.8 \times 10^{6}$ \\
14 & $3.3 \times 10^{7}$ \\
\hline \hline
\end{tabular}

analytical expressions of the higher orders become lengthy and complex, if one focuses on the expression for the transmitted field only, it is found that the amplitude of the field increases with the product $\Delta \varepsilon_{r} d$. The ratio between the second and first perturbation orders is of the order of $\left|\Delta \varepsilon_{r} d \pi /(2 \lambda)\right|$, which can be taken as an indication of the transition between the weak- and strong-scattering regime: if $\left|\Delta \varepsilon_{r} d \pi /(2 \lambda)\right|>1$ the series diverges. It is easy to check that for the wavelength and thickness $d$ and all the chosen permittivity contrasts $\Delta \varepsilon_{r}$ in this work the series in Eq. (6) indeed diverges. For instance, for the case $\varepsilon_{r}=4$, the absolute value of the fifteenth term of the series reaches the value of order $1 \times 10^{7}$, which clearly indicates strong divergence. To be more specific, in Table I, we report the absolute value of the field at the interface at $z=1.5 \mu \mathrm{m}$ of most of the first fifteen perturbation terms of the series in Eq. (6). Some of the perturbation terms are shown in Fig. 2, where the divergence of the series is evident. It is clear that, because of the strong divergence of the series, simply adding up all terms as suggested by Eq. (6) cannot lead to any meaningful estimate of the total field $U(z)$. The divergent behavior of a perturbation series is surely disappointing, especially considering the effort required to compute even only a few of the perturbation terms. On the other hand, no convergence issues are encountered for a Born series that operates within the weak-scattering regime, which are however much less interesting from the perspective of applications in imaging and superresolution due to the fact that multiplescattering phenomena are negligible in that case [22-24]. By following the approach using the Padé approximant described in Sec. II, we were able to provide an accurate approximation of the field. Fig. 3(a) shows (the absolute value of) the Padé approximant $P_{6}^{6}(z)$ for all three cases included in Fig. 1.

As evident from the figure, a very accurate approximation of the analytical solution is obtained. Fig. 3(b) shows how the convergence to the solution is reached by the Padé rational polynomials. Finally, in Table II, we show the values of the Padé approximants $P_{0}^{0}(z)$ to $P_{6}^{6}(z)$ at $z=1.5 \mu \mathrm{m}$.

As evident from the table, an accuracy of $0.0030 \%$ and $0.16 \%$ is reached for the real and imaginary part of the field, respectively. Which order of Padé approximants should be used to obtain an accurate estimate of the field depends on 

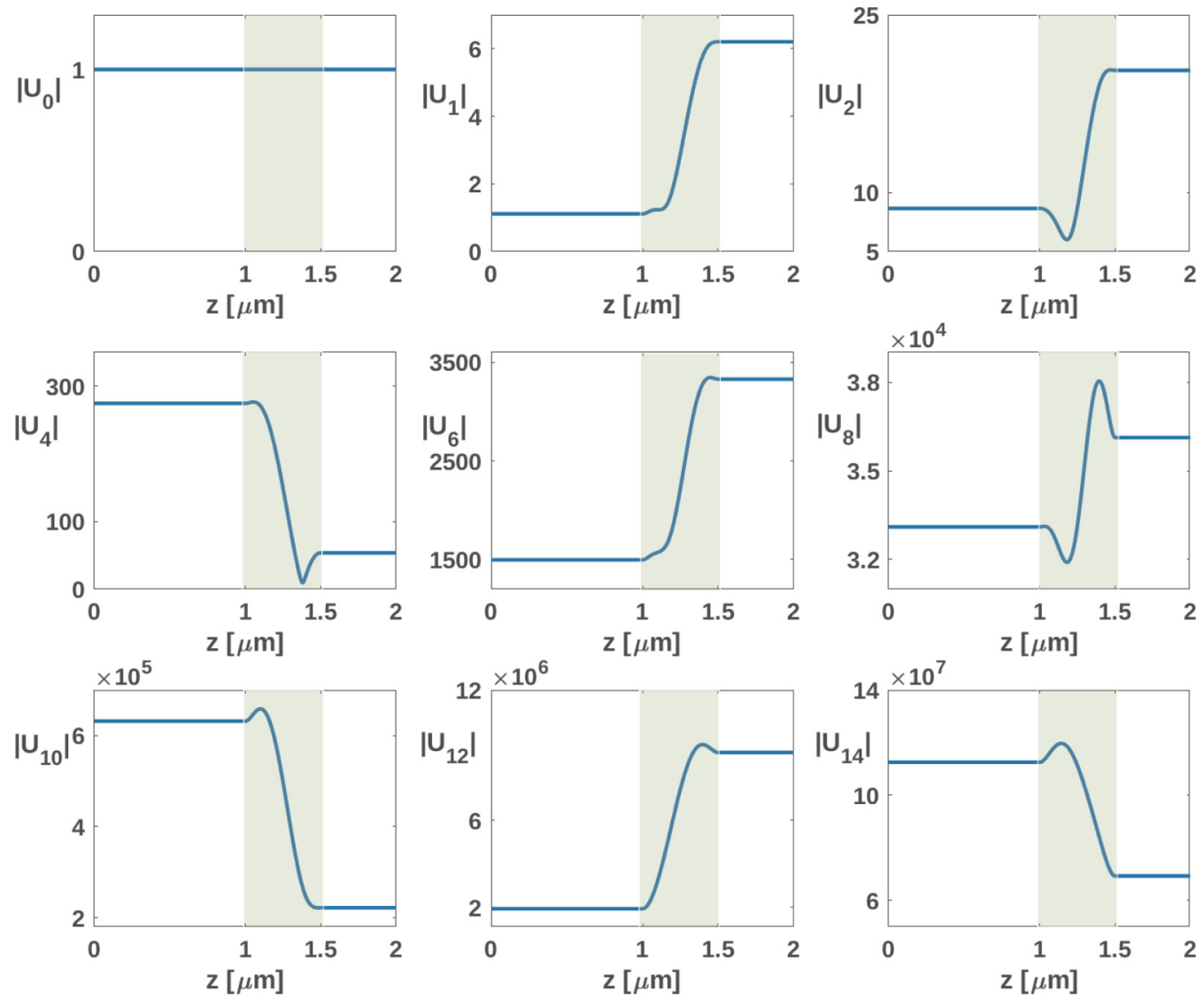

FIG. 2. Some perturbation orders of Eq. (6), for the case where $\varepsilon_{r}=4+i, d=500 \mathrm{~nm}$ and $\lambda=800 \mathrm{~nm}$. The series clearly diverges for any value of $z$.

different factors, such as the permittivity contrast $\Delta \varepsilon_{r}$ and how important multiple scattering is in the problem under study. For the cases discussed here, the solution saturates at $P_{6}^{6}(z)$ since the relative difference between $P_{5}^{5}(z)$ and $P_{6}^{6}(z)$ is of the same order as between $P_{6}^{6}(z)$ and the (in this case known) analytical solution. This makes approximants of higher orders ineffective and unnecessary.

\section{STRONG ELECTROMAGNETIC SCATTERING REGIME: TWO-DIMENSIONAL CASE}

In this section, we turn our attention to two-dimensional problems. We will consider the scattering of an incident field by an infinitely long cylinder of radius $R$ because, also for

TABLE II. Value of Padé approximants from 0th to 6th order, for $\varepsilon_{r}=4, \lambda=800 \mathrm{~nm}$ at position $z=1.5 \mu \mathrm{m}$. The correct value of the total field is $U(z=1.5 \mu \mathrm{m})=-0.800000000+0 i$.

\begin{tabular}{lc}
\hline \hline Padé order $N$ & Value Padé \\
\hline 0 & $0.707106781-\mathrm{i} 0.707106781$ \\
1 & $0.225282626+\mathrm{i} 0.924861295$ \\
2 & $-0.209472028+\mathrm{i} 0.746123727$ \\
3 & $-0.506689994-\mathrm{i} 4.29529490 \times 10^{-2}$ \\
4 & $-0.834428691+\mathrm{i} 3.89980981 \times 10^{-3}$ \\
5 & $-0.799387484+\mathrm{i} 1.61243988 \times 10^{-3}$ \\
6 & $-0.799975820+\mathrm{i} 1.67074306 \times 10^{-3}$ \\
\hline \hline
\end{tabular}

this case, an analytical solution exists. The incident field consists of a plane wave, with the direction of propagation perpendicular to the axis of the cylinder. The background permittivity is again that of vacuum $\varepsilon_{0}$, while the cylinder is homogeneous and made of an isotropic material with (possibly complex) relative electric permittivity $\varepsilon_{r}$. Hence, the permittivity contrast $\Delta \varepsilon_{r}=\varepsilon_{r}-1$ is constant within the cylinder and zero outside. We choose a Cartesian coordinate system such that the $z$ axis coincides with the axis of the cylinder. If the electric and magnetic fields, $\mathbf{E}$ and $\mathbf{H}$, do not depend on $z$, i.e., if the incident field is two-dimensional, it can be shown from the source-free Maxwell's equations that two uncoupled Helmholtz equations can be obtained, one for $E_{z}$ and one for $H_{z}$. The boundary conditions for the fields are then uncoupled as well. Therefore, two polarizations states, TM and TE, can be distinguished, where $E_{z}$ and $H_{z}$ are parallel to the $z$ axis, respectively. Here, we will consider TM polarization and we choose the incident field to be a plane wave with $E_{z}(x, y)=U_{i}(x, y)=\exp \left[i k_{0} x\right]$, which propagates in the positive $x$ direction, as shown in Fig. 4. In TM polarization, $E_{z}$ satisfies the equation

$$
\nabla^{2} E_{z}(x, y)+k_{0}^{2} \varepsilon_{r}(x, y) E_{z}(x, y)=0,
$$

both inside and outside of the cylinder and $E_{z}$ and the normal derivative $\partial E_{z} / \partial r$ are both continuous at the boundary $r=R$ 
(a)

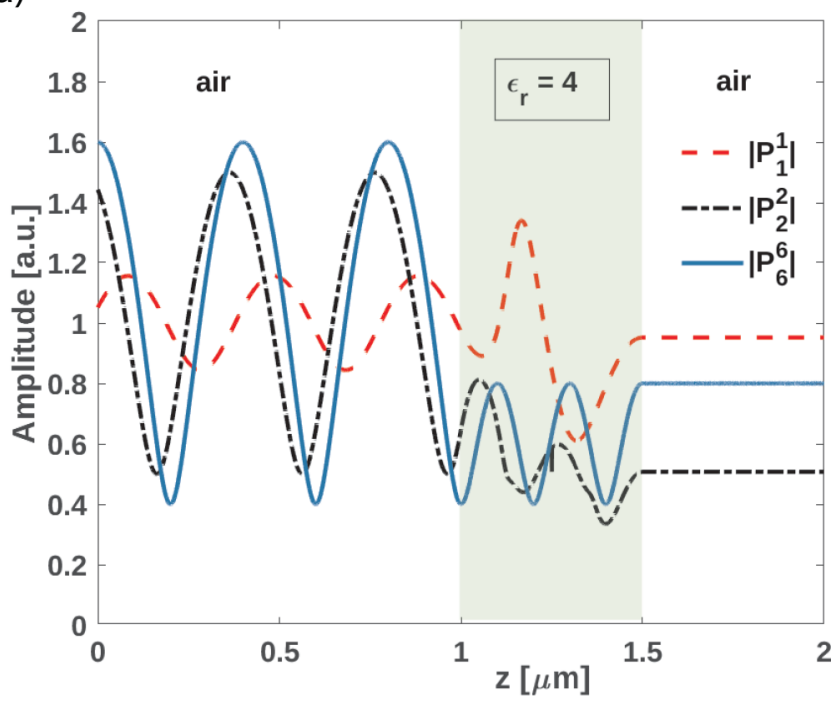

(b)

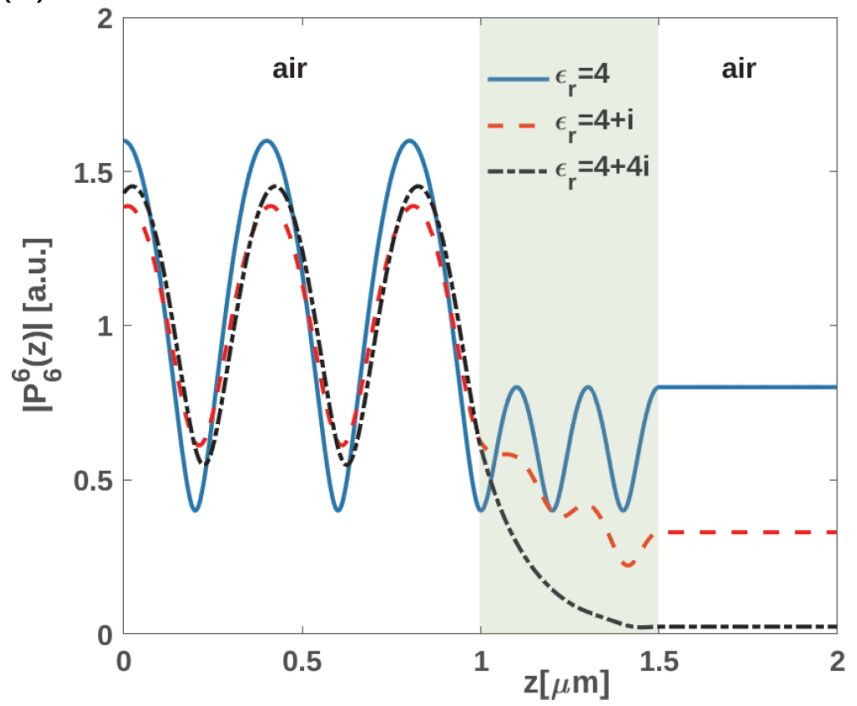

FIG. 3. Approximation of the total field as obtained through Padé rational functions. (a) shows the approximation of the solution of the scattering problem by using the Padé approximants. The figure shows the absolute value of the $P_{6}^{6}(z)$ rational polynomial for the three different cases given in Fig. 1. Comparing the plots to Fig. 1, it is evident that the Padé approximants provide an accurate estimate of the solution. (b) shows the convergence to the analytical solution as reached by Padé rational functions for a medium with $\varepsilon_{r}=4$. The Padé approximants $P_{1}^{1}(z), P_{3}^{3}(z)$ and $P_{6}^{6}(z)$ are shown.

of the cylinder. Here, $\varepsilon_{r}(x, y)$ is a scalar field

$$
\varepsilon_{r}(x, y)=\left\{\begin{array}{ll}
\varepsilon_{r}, & x^{2}+y^{2}<R \\
1, & x^{2}+y^{2}>R
\end{array} .\right.
$$

The terms in the Born series and the Padé approximants are computed as explained in Sec. II, but now with $G\left(\mathbf{r}, \mathbf{r}^{\prime}\right)$ given by the 2D free-space Green's function:

$$
G\left(\mathbf{r}, \mathbf{r}^{\prime}\right)=\frac{i}{4} H_{0}^{(1)}\left(k_{0}\left|\mathbf{r}-\mathbf{r}^{\prime}\right|\right) .
$$

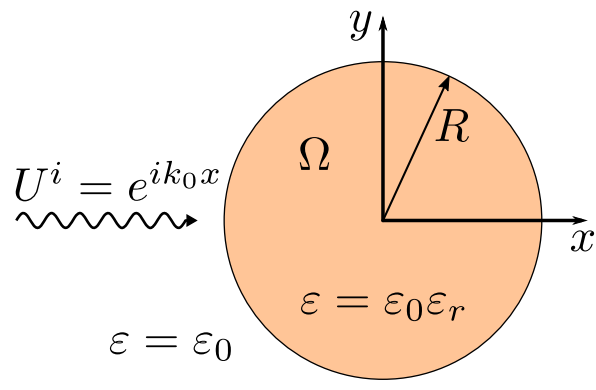

FIG. 4. Geometry of the $2 \mathrm{D}$ problem. The incident plane wave $U_{i}$ represents either the $z$ component of the electric field, $E_{z}$, or the $z$ component of the magnetic field $H_{z}$. In this work, we will only consider the TM polarization case (namely the $E_{z}$ case).

The analytical solution to the Helmholtz equation for $E_{z}$ can be found by writing the Helmholtz equation in polar coordinates $(r, \varphi)$ and expanding $E_{z}$ in a Fourier series as a function of $\varphi$. For more details, the reader can refer to Appendix D. Also for the 2D case, in order to assess the performance of the method, we consider the scattering from different cylinders, namely a metal and a semiconductor cylinder with relative permittivities of silver and silicon, which at the wavelength $\lambda=400 \mathrm{~nm}$ are given by $\varepsilon_{r, \mathrm{Ag}}=-4.42+0.201 i$ [27] and $\varepsilon_{r, \mathrm{Si}}=30.8+4.30 i$ [28], respectively. For each material, we perform the calculations for two cylinder radii, $100 \mathrm{~nm}$ and $400 \mathrm{~nm}$, so four different configurations are investigated, one for each combination of size and permittivity. The cases are listed in Table III. In the same table, we also show the values of two parameters, $\Gamma_{\mathrm{Born}}^{(a)}$ and $\Gamma_{\mathrm{Born}}^{(n)}$ which are introduced to have a useful metric to indicate when a Born series starts to diverge. We define:

$$
\Gamma_{\text {Born }}^{(a)}=\frac{\pi R\left|\Delta \varepsilon_{r}\right|}{\lambda} .
$$

Like in the 1D case, it is found by taking the absolute value of the ratio between the second and first perturbation orders and serves as an indication of the transition between the weakand strong-scattering regime. The Born series converges or diverges when $\Gamma_{\text {Born }}^{(a)}<1$ or $\Gamma_{\text {Born }}^{(a)}>1$, respectively. This criterion turns out to be in good agreement with that derived for the 1D case, if one considers that now $R$ is half the diameter of the scatterer. Next to this, the parameter $\Gamma_{\mathrm{Born}}^{(n)}$ is given by

$$
\Gamma_{\text {Born }}^{(n)}=\left(\frac{A_{\ell_{\max }}}{A_{0}}\right)^{\frac{1}{\varepsilon_{\max }}}
$$

TABLE III. Specifications of the four simulated configurations in the $2 \mathrm{D}$ case.

\begin{tabular}{lccccc}
\hline \hline Case & \multirow{2}{*}{ Material } & $\varepsilon_{r}$ & $R(\mathrm{~nm})$ & $\Gamma_{\text {Born }}^{(a)}$ & $\Gamma_{\text {Born }}^{(n)}$ \\
\hline I & $\mathrm{Ag}$ & $-4.42+\mathrm{i} 0.201$ & 100 & 4.26 & 5.67 \\
II & $\mathrm{Si}$ & $30.8+\mathrm{i} 4.30$ & 100 & 23.7 & 31.5 \\
III & $\mathrm{Ag}$ & $-4.42+\mathrm{i} 0.201$ & 400 & 17.0 & 14.9 \\
IV & $\mathrm{Si}$ & $30.8+\mathrm{i} 4.30$ & 400 & 94.7 & 83.0 \\
\hline \hline
\end{tabular}




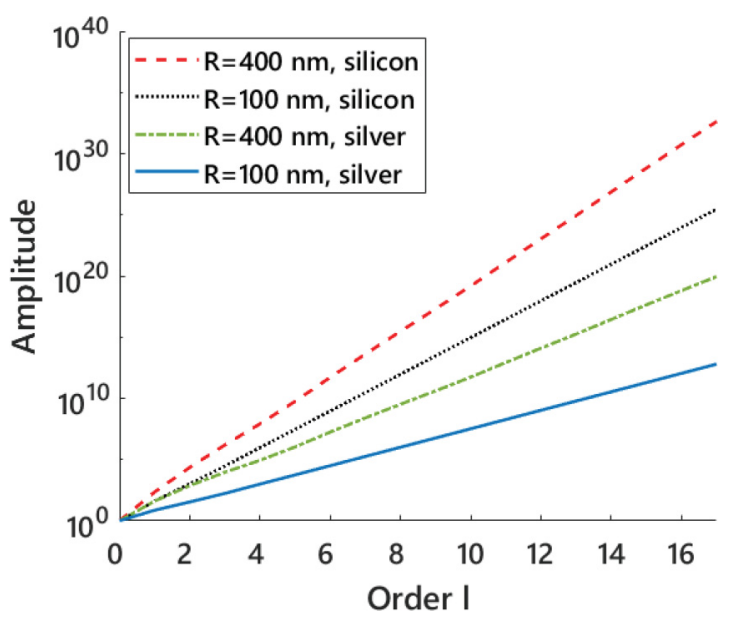

FIG. 5. Amplitude of the $\ell$-th term of the Born series, from 0th to 17 th order, for cylinders of different sizes and materials. In all cases, the terms show an exponential growth.

where $\ell_{\max }$ is the highest order of the calculated Born terms, $A_{\ell}=\max _{\mathbf{r} \in \Omega}\left|U_{\ell}(\mathbf{r})\right|$ is the maximum of the absolute value of the Born term of order $\ell$ and $\Omega$ is the domain of the cylinder. $A_{0}=1$ for all cases since the incident plane wave has amplitude 1 . The parameter $\Gamma_{\mathrm{Born}}^{(n)}$ is determined from the numerical calculations of the Born terms and therefore gives a more realistic indication of the divergence of the series compared to Eq. (13). The values of $\Gamma_{\text {Born }}^{(a)}$ and $\Gamma_{\text {Born }}^{(n)}$ are listed in Table III for each configuration considered. It is readily seen that all considered configurations are far into the strong-scattering regime. Numerical calculations of the Born terms indeed confirm that in all four cases the series diverges strongly. In Fig. 5, we show the maximum (over all points inside the scatterer) of the absolute value of the field per Born term. Since we also calculate the analytical solution, the quality of the solution obtained through Padé approximants can be assessed by determining the relative error of the Padé approximants, using the total electric energy inside the cylinder as norm,

$$
e_{N}=\frac{\iint_{\Omega} \operatorname{Re}\left(\varepsilon_{r}\right)\left|E_{z, \text { analytical }}-P_{N}^{N}\right|^{2} d x d y}{\iint_{\Omega} \operatorname{Re}\left(\varepsilon_{r}\right)\left|E_{z, \text { analytical }}\right|^{2} d x d y} .
$$

Also, we define the absolute error of a Padé approximant per point, as compared to the analytical solution, as follows:

$$
e_{\mathrm{abs}, N}(\mathbf{r})=\left|E_{z, \text { analytical }}(\mathbf{r})-P_{N}^{N}(\mathbf{r})\right| .
$$

With the relative error $e_{N}$, we selected the best Padé approximant of $E_{z}$ for each of the analyzed cases. In Fig. 6, the analytical solution is shown together with the Padé approximant for which error (15) is minimum, together with the absolute error (16). Also, a plot of $e_{N}$ as a function of the order $N$ of the approximant is included. For all cases, we observe that the best Padé approximant approximates the analytical solution very well, though the error increases for higher values of $\Gamma_{\mathrm{Born}}^{(a)}$ as expected. For instance, the best approximant for case I is $P_{5}^{5}$. Nevertheless, $P_{5}^{5}$ approximates the analytical solution also in this case quite accurately although $\Gamma_{\text {Born }}^{(a)}=4.26$ and hence this is well inside the strong-scattering regime. The maximum of the absolute error $e_{\mathrm{abs}, 5}(\mathbf{r})$ is $1.3 \times 10^{-4}$. Even for the configuration with the highest $\Gamma_{\mathrm{Born}}^{(n)}$, case IV, the Padé approximant $P_{11}^{11}$ is still able to retrieve $E_{z}$ from the diverging Born series. In order to compute $P_{11}^{11}, \ell_{\max }=22$ terms of the Born series are needed. Remarkably, there is a difference between the magnitude of the lowest and the highest order Born term, $A_{0}$ and $A_{22}$, of order $1 \times 10^{42}$. In the majority of cases, the largest pointwise errors [Eq. (16)] are found near or within the scattering cylinder. From the plots of $e_{N}$, we observe that for increasing order $N$ of the approximant, the error decreases monotonically initially. However, from a certain order, the approximants start to show numerical instabilities and artifacts start to dominate, which have noisy features. The order $N$ at which this happens, appears to depend mostly on the radius of the cylinder relative to the wavelength. A larger permittivity worsens the amplitude of the noisy features. In stable Padé approximants, often linelike artifacts arising from the cylinder can be observed, as in panel (f) of Fig. 6 for instance. In this 2D case (as well as in the 1D case), the analytical solution can be used to determine which Padé approximant is the most accurate. However, for complex geometries where no analytical solution is available, the best approximant has to be found differently. For instance, one could calculate the difference between two approximants of consecutive order $N-1$ and $N$ and determine where the difference is at a minimum. That would point to a stagnation in improvement of the approximations, since at a certain order $N$ the approximants start to destabilize and the approximations deteriorate quickly. Quantifying this, it would correspond to finding the $N_{\text {best }}$ for which $e_{\text {consec }, N}=\iint\left|P_{N}^{N}-P_{N-1}^{N-1}\right|^{2} d x d y$ is minimal. This approach actually returns the same $N$ for cases I, II, and III as when comparing it to the analytical solution. For case IV, this approach gives $N_{\text {best }}=12$ instead of $N=11$.

\section{CONCLUSIONS}

In this paper, an accurate approximation of the electromagnetic field was derived from the Born series which can be used even under strong-scattering conditions. The divergence of the Born series under these conditions can be cured using the Padé method and very accurate results can be obtained in this way. We have explicitly analyzed $1 \mathrm{D}$ and $2 \mathrm{D}$ cases, where analytical solutions are available. In this way, it was possible to quantify the accuracy of the approximations found. It is important to point out that the dimensionality of the problem only affects the way the term of the Born series are computed, not the way Padé approximants are derived from the terms of the Born series. In this sense, the areas of applicability of the methods described here are broad and expected to have impact on different areas of theoretical and applied electromagnetic problems. One main advantage of the approach is that the various terms of the original series are used as they are. In fact, the physical meaning of the higher-orders scattering processes is preserved, which is an advantage of using the Born series to solve a scattering problem. We expect that the physical insights provided by the terms of the Born series can result in a powerful tool for inverse scattering problems as well. We will report on some of these applications in a future work. 
(a)

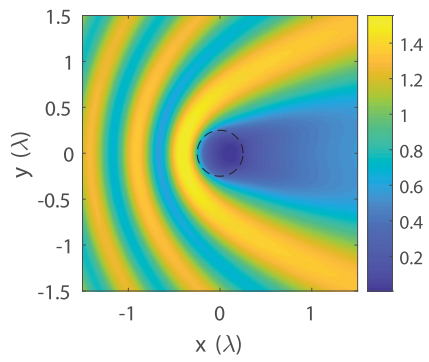

(e)

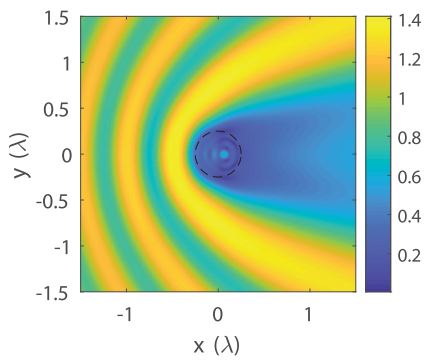

(i)

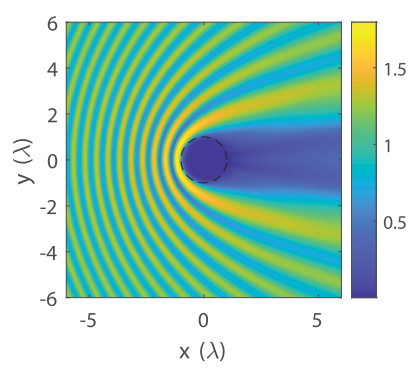

(m)

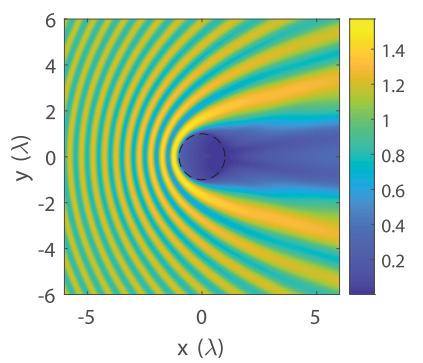

(b)

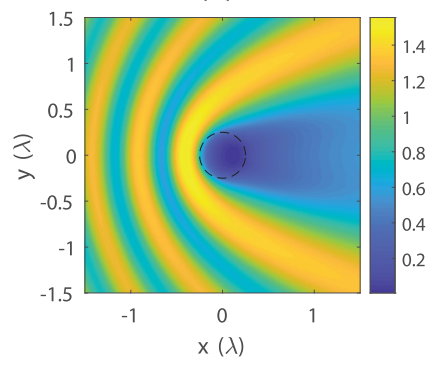

(f)

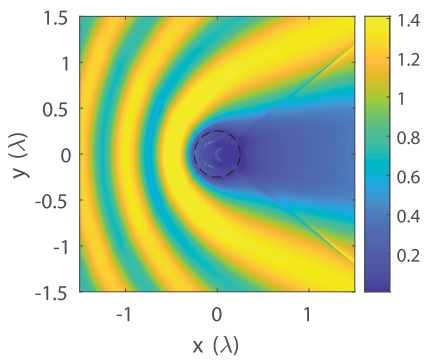

(j)

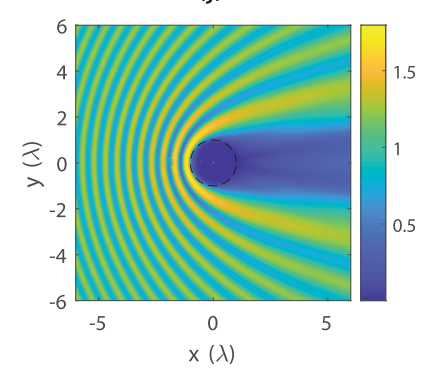

(n)

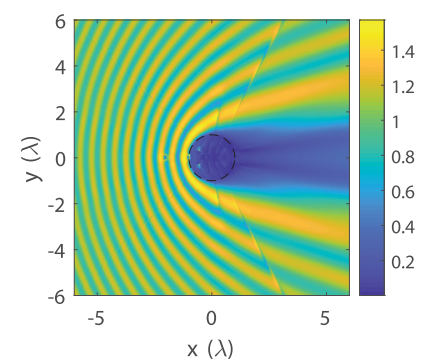

(c)

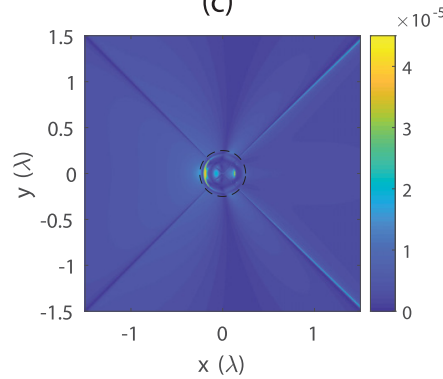

(g)

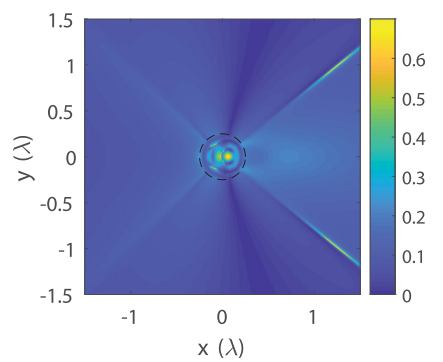

(k)

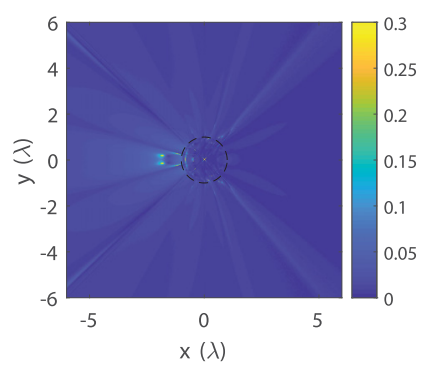

(o)

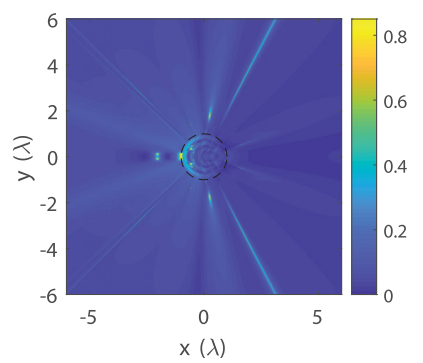

(d)

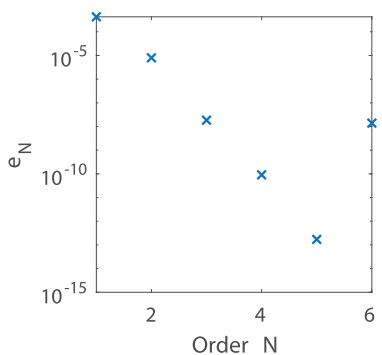

(h)

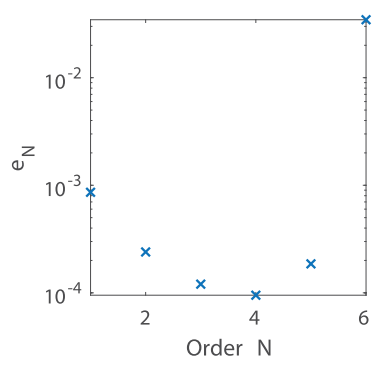

(l)

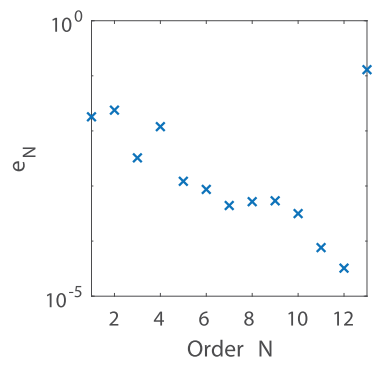

(p)

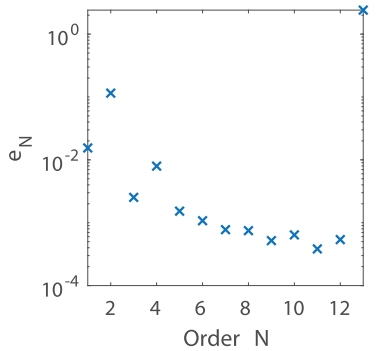

FIG. 6. Analytical solution, the best Padé approximant, the error $e_{\mathrm{abs}, N}$ of that approximant and the relative error per Padé order for four different configurations. (a)-(d) concern a silver cylinder with $R=100 \mathrm{~nm}$. (a) shows the analytical solution, (b) the Padé approximant $P_{5}^{5}$, (c) the absolute error $e_{\mathrm{abs}, 5}(\mathbf{r})$, and (d) the relative energy error per Padé order. (e)-(h) concern a silicon cylinder with $R=100 \mathrm{~nm}$. (e) shows the analytical solution, (f) the Padé approximant $P_{4}^{4},(\mathrm{~g})$ the absolute error $e_{\text {abs, } 4}(\mathbf{r})$, (h) the relative energy error per Padé order. (i)-(l) concern a silver cylinder with $R=400 \mathrm{~nm}$. (i) shows the analytical solution, (j) the Padé approximant $P_{12}^{12}$, (k) the absolute error $e_{\mathrm{abs}, 12}(\mathbf{r})$, and (l) the relative energy error per Padé order. (m)-(p) concern a silicon cylinder with $R=400 \mathrm{~nm}$. (m) shows the analytical solution, (n) the Padé approximant $P_{11}^{11}$, (o) the absolute error $e_{\mathrm{abs}, 11}(\mathbf{r})$, and (p) the relative energy error per Padé order. The black dashed circle shows the boundary of the cylinder.

\section{ACKNOWLEDGMENTS}

This work was funded through the EMPIR project 17FUN01-BeCOMe. The EMPIR initiative is co-funded by the European Union Horizon 2020 research and innovation programme and the EMPIR participating States.

O.E.G. and H.P.U. have initiated this research, worked out the theoretical framework and supervised the work. T.A.v.d.S. 
has fully implemented the 2D case. All authors have contributed to the writing.

\section{APPENDIX A: COMPUTING PADÉ APPROXIMANTS}

In this Appendix, we will show how to compute the symmetric Padé approximant

$$
P_{N}^{N}(\sigma, \mathbf{r})=\frac{\sum_{n=0}^{N} A_{n}^{(N)}(\mathbf{r}) \sigma^{n}}{1+\sum_{m=1}^{N} B_{m}^{(N)}(\mathbf{r}) \sigma^{m}} .
$$

The approximant of order $N$ has $2 N+1$ unknowns that can be determined by equating $P_{N}^{N}$ to the first $2 N+1$ terms of the Born series. We will explicitly show how to do this for $N=0,1,2$ and we will also provide the general formula for higher $N$. Throughout this Appendix the dependence on $\mathbf{r}$ in the equations will be omitted for brevity.

Case $N=0$. For the case of $N=0$, we only need the first term in the Born series, which is just the incident field.

$$
P_{0}^{0}(\sigma)=A_{0}^{(0)}=U_{0}=U_{i},
$$

Case $N=1$. Now we have three unknowns $A_{0}^{(1)}, A_{1}^{(1)}$, and $B_{1}^{(1)}$ in the approximant

$$
P_{1}^{1}(\sigma)=\frac{A_{0}^{(1)}+A_{1}^{(1)} \sigma}{1+B_{1}^{(1)} \sigma} .
$$

Equating this to the first three terms of the Born series and rewriting the equation by collecting the terms with equal powers of $\sigma$ gives an equation for each power of $\sigma$, resulting in a system of three equations

$$
\begin{aligned}
A_{0}^{(1)} & =U_{0}, \\
A_{1}^{(1)} & =U_{1}+U_{0} B_{1}^{(1)}, \\
0 & =U_{2}+U_{1} B_{1}^{(1)} .
\end{aligned}
$$

First we can solve for $B_{1}^{(1)}$ and with the result we calculate $A_{1}^{(1)}$, yielding

$$
\begin{aligned}
& B_{1}^{(1)}=-\frac{U_{2}}{U_{1}}, \\
& A_{0}^{(1)}=U_{0}, \\
& A_{1}^{(1)}=U_{1}-U_{0} \frac{U_{2}}{U_{1}} .
\end{aligned}
$$

The Padé approximant therefore becomes

$$
\begin{aligned}
P_{1}^{1}(\sigma=1) & =\frac{U_{0}+U_{1}-U_{0} \frac{U_{2}}{U_{1}}}{1-\frac{U_{2}}{U_{1}}} \\
& =U_{0}+U_{1} \frac{U_{1}}{U_{1}-U_{2}} .
\end{aligned}
$$

Case $N=2$. Similarly, for $N=2$, we have a system of equations

$$
\begin{aligned}
A_{0}^{(2)} & =U_{0}, \\
A_{1}^{(2)} & =U_{1}+U_{0} B_{1}^{(2)}, \\
A_{2}^{(2)} & =U_{2}+U_{1} B_{1}^{(2)}+U_{0} B_{2}^{(2)} \\
0 & =U_{3}+U_{2} B_{1}^{(2)}+U_{1} B_{2}^{(2)}, \\
0 & =U_{4}+U_{3} B_{1}^{(2)}+U_{2} B_{2}^{(2)},
\end{aligned}
$$

of which we first solve Eqs. (A7d) and (A7e) for $B_{m}^{(2)}$. We can use Cramer's rule for the system $T x=b$, where

$$
T=\left(\begin{array}{ll}
U_{2} & U_{1} \\
U_{3} & U_{2}
\end{array}\right), \quad b=\left(\begin{array}{c}
-U_{3} \\
-U_{4}
\end{array}\right) \quad x=\left(\begin{array}{l}
B_{1}^{(2)} \\
B_{2}^{(2)}
\end{array}\right) .
$$

According to the rule, $B_{m}^{(2)}=\operatorname{det}\left(T_{m}\right) / \operatorname{det}(T)$, where $T_{m}$ is obtained by replacing the $m$ th column of $T$ with $b$. We define $\Delta \equiv \operatorname{det}(T)$ and $\Delta_{m} \equiv \operatorname{det}\left(T_{m}\right)$. We get

$$
\begin{aligned}
& B_{1}^{(2)}=\frac{\Delta_{1}}{\Delta}=\frac{-U_{3} U_{2}+U_{4} U_{1}}{U_{2}^{2}-U_{3} U_{1}}, \\
& B_{2}^{(2)}=\frac{\Delta_{2}}{\Delta}=\frac{-U_{2} U_{4}+U_{3}^{2}}{U_{2}^{2}-U_{3} U_{1}},
\end{aligned}
$$

which we can then plug in Eqs. (A7a)-(A7c) to find $A_{n}^{(2)}$. For $P_{2}^{2}$ we obtain, rewriting the terms

$$
\begin{aligned}
P_{2}^{2}(\sigma=1) & =\frac{A_{0}^{(2)}+A_{1}^{(2)}+A_{2}^{(2)}}{1+B_{1}^{(2)}+B_{2}^{(2)}} \\
& =U_{0}+U_{1} \frac{\Delta+\Delta_{1}}{\Delta+\Delta_{1}+\Delta_{2}}+U_{2} \frac{\Delta}{\Delta+\Delta_{1}+\Delta_{2}} .
\end{aligned}
$$

General case. For any order $\mathrm{N}$, the coefficients $A_{n}^{(N)}$ and $B_{m}^{(N)}$ in $P_{N}^{N}$ can be found by equating $P_{N}^{N}$ to the first $2 N+1$ terms of the Born series. This results in a system of $2 N+1$ equations, of which a system of $N$ equations can be solved with Cramer's rule (where $\Delta$ and $\Delta_{m}$ are the determinants) to find the $B_{m}^{(N)}$ coefficients. Then the other $N+1$ equations are used to find the $A_{m}^{(N)}$ coefficients. As a result

$$
\begin{gathered}
B_{m}^{(N)}=\frac{\Delta_{m}}{\Delta}, \\
A_{n}^{(N)}=U_{n}+\sum_{\ell=0}^{n-1} U_{\ell} B_{n-\ell}=U_{n}+\sum_{\ell=0}^{n-1} U_{\ell} \frac{\Delta_{\ell}}{\Delta},
\end{gathered}
$$

such that

$$
\begin{aligned}
P_{N}^{N}(\sigma) & =\frac{\sum_{n=0}^{N} A_{n}^{(N)} \sigma^{n}}{1+\sum_{m=1}^{N} B_{m}^{(N)} \sigma^{m}} \\
& =\sum_{\ell=0}^{N} U_{\ell} \sigma^{\ell} \frac{\Delta+\sum_{m=1}^{N-\ell} \Delta_{m} \sigma^{m}}{\Delta+\sum_{m=1}^{N} \Delta_{m} \sigma^{m}} .
\end{aligned}
$$

Setting $\sigma=1$, we arrive at

$$
P_{N}^{N}(\sigma=1)=\sum_{\ell=0}^{N} U_{\ell} \frac{\Delta+\sum_{m=1}^{N-\ell} \Delta_{m}}{\Delta+\sum_{m=1}^{N} \Delta_{m}} .
$$

\section{APPENDIX B: ANALYTICAL SOLUTION TO THE 1D PROBLEM}

In this Appendix, we will provide some more details on the expressions of the analytical solutions to the 1D problem used to assess the performances of the methods presented in this work. The reader can refer to Fig. 1 for the geometry. We start by expressing the total field as $U(z)=U_{i}(z)+U_{s}(z)$ and we know that it must satisfy the 1D Helmholtz equation $\partial_{z}^{2} U(z)+$ $k_{0}^{2} U(z)=0$ in the three domains $z<z_{1}, z_{1} \leqslant z \leqslant z_{1}+d$ and 
$z>z_{1}+d$. The solutions are found by imposing the boundary conditions for the field at the two interfaces. If we denote the amplitude of the permittivity contrast by $\Delta \varepsilon_{r}$, the refractive index by $n=\sqrt{\Delta \varepsilon_{r}}$ and plane waves by $\psi_{ \pm}=\exp \left[ \pm i k_{0} z\right]$, the analytical solution of this problem reads

$$
U(z)= \begin{cases}\psi_{+}+a_{1}^{-} \psi_{-}, & \text {for } z<z_{1} \\ a_{2}^{+} \psi_{+}+a_{2}^{-} \psi_{-}, & \text {for } z_{1} \leqslant z \leqslant z_{1}+d \\ a_{3}^{+} \psi_{+}, & \text {for } z>z_{1}+d\end{cases}
$$

with the coefficients $a_{1}^{-}, a_{2}^{+}, a_{2}^{-}$and $a_{3}^{+}$being defined as

$$
\begin{gathered}
a_{1}^{-}=\frac{1}{D}\left[-C^{+} i k_{0} \exp \left[i k_{0}(n+1) z_{1}\right](n-1)\right. \\
+C^{-} i k_{0} \exp \left[-i k_{0}(n-1) z_{1}\right](n+1), \\
a_{2}^{ \pm}=a_{3}^{+} C^{ \pm}, \\
a_{3}^{+}=\frac{2 i k_{0}}{D},
\end{gathered}
$$

where the coefficients $C^{+}, C^{-}$and $D$ have expressions

$$
\begin{aligned}
C^{ \pm} & =\frac{\exp \left[i k_{0}(1 \mp n)\left(z_{1}+d\right)\right](n \pm 1)}{2 n} \\
D= & C^{+} i k_{0}(n+1) \exp \left[+i k_{0}(n-1) z_{1}\right] \\
& -C^{-} i k_{0}(n-1) \exp \left[-i k_{0}(n+1) z_{1}\right] .
\end{aligned}
$$

\section{APPENDIX C: PERTURBATION ORDERS OF THE 1D PROBLEM}

As explained in the main manuscript, because analytical solutions are rarely available, it is important to find ways to solve the same problem through the perturbation series approach. This means expressing the solution in the following way:

$$
U^{(\sigma)}(\mathbf{r})=\sum_{\ell=0}^{\infty} U_{\ell}(\mathbf{r}) \sigma^{\ell} .
$$

While all terms of such series can be computed numerically, in this work we are also providing the analytical expression of the first three terms of the series. This is useful because one can validate the numerical calculations used to compute all terms of the series by comparing numerical results with those analytical expressions. In addition to that, an analytical expression of a few orders of the perturbation series helps to identify the transition between the weak- and strongscattering regime and which physical parameters control such transition. For the specific case studied in this work, we provide the expressions of the first three terms of the Born series, $U_{0}(z), U_{1}(z)$, and $U_{2}(z)$, in Eqs. (C2)-(C4) in the case the incident field is a plane wave $U_{i}(z)=\exp \left(i k_{0} z\right)$. We use the notation $\psi_{ \pm}=\exp \left( \pm i k_{0} z\right)$ to denote plane waves.

$U_{0}(z)=\exp \left(i k_{0} z\right) \quad$ for all $z$.

$$
\begin{gathered}
U_{1}(z)=\frac{\Delta \varepsilon_{r}}{4} \begin{cases}\psi_{-} \exp \left(2 i k_{0} z_{1}\right)\left[\exp \left(2 i k_{0} d\right)-1\right] & \text { for } z<z_{1}, \\
\psi_{+}\left[2 i k_{0}\left(z-z_{1}\right)-1\right]+\psi_{-} \exp \left[2 i k_{0}\left(d+z_{1}\right)\right] & \text { for } z_{1} \leqslant z \leqslant z_{1}+d, \\
\psi_{+} 2 i k_{0} d & \text { for } z>z_{1}+d,\end{cases} \\
U_{2}(z)=\frac{\Delta \varepsilon_{r}^{2}}{16} \begin{cases}\psi_{-} \exp \left(2 i k_{0} z_{1}\right)\left\{2 i k_{0} d \exp \left(2 i k_{0} d\right)-\left[\exp \left(2 i k_{0} d\right)-1\right]\right\} & \text { for } z<z_{1}, \\
\psi_{+}\left\{\left[1+\frac{1}{2} \exp \left(2 i k_{0} d\right)\right]-2 i k_{0}\left(z-z_{1}\right)-k_{0}^{2}\left(z-z_{1}\right)^{2}\right\} & \\
+\psi_{-} \exp \left[2 i k_{0}\left(z_{1}+d\right)\right]\left[-\frac{3}{2}+i k_{0}\left(z_{1}+2 d-z\right)\right] & \text { for } z_{1} \leqslant z \leqslant z_{1}+d, \\
\psi_{+}\left\{-\frac{1}{2}\left[1-\exp \left(2 i k_{0} d\right)\right]-i k_{0} d-k_{0}^{2} d^{2}\right\} & \text { for } z>z_{1}+d .\end{cases}
\end{gathered}
$$

\section{APPENDIX D: ANALYTICAL SOLUTION TO THE 2D PROBLEM}

The analytical solution of $E_{z}$ in the 2D case, which satisfies Eq. (10), is presented in this Appendix. The derivation can be found in electromagnetic theory textbooks such as [29-31], although the notations might differ. Also [32] provides a general solution for incoming plane waves under any angle. The solution is given as a Fourier series of $E_{z}(r, \varphi)$ as a function of $\varphi$ :

$$
E_{z}(r, \varphi)=\sum_{m=-\infty}^{+\infty} \hat{E}_{z}(r, m) \exp (\operatorname{im} \varphi)
$$

where $\hat{E}_{z}(r, m)$ are the Fourier coefficients. The analytical solution is:

$$
\hat{E}_{z}(r, m)= \begin{cases}A_{m} J_{m}\left(k_{0} r\right)+B_{m} H_{m}^{(1)}\left(k_{0} r\right) & \text { for } r>R, \\ C_{m} J_{m}\left(k_{1} r\right) & \text { for } r<R,\end{cases}
$$

where $J_{m}$ and $H_{m}^{(1)}$ are the Bessel and the Hankel function of the first kind and $k_{1}=k_{0} \sqrt{\varepsilon_{r}}$. The coefficients $A_{m}$ are determined by the incident field, which for a plane wave are $A_{m}=i^{m}$. The coefficients $B_{m}$ and $C_{m}$ (corresponding to the scattered field and field inside the cylinder, respectively) are then given in terms of $A_{m}$ by Eq. (D3).

$$
\begin{aligned}
& B_{m}=-\frac{J_{m}\left(k_{1} R\right) J_{m+1}\left(k_{0} R\right)-\sqrt{\varepsilon_{r}} J_{m+1}\left(k_{1} R\right) J_{m}\left(k_{0} R\right)}{J_{m}\left(k_{1} R\right) H_{m+1}^{(1)}\left(k_{0} R\right)-\sqrt{\varepsilon_{r}} J_{m+1}\left(k_{1} R\right) H_{m}^{(1)}\left(k_{0} R\right)} A_{m}, \\
& C_{m}=\frac{-2 i}{\pi k_{0} R} \frac{1}{J_{m}\left(k_{1} R\right) H_{m+1}^{(1)}\left(k_{0} R\right)-\sqrt{\varepsilon_{r}} J_{m+1}\left(k_{1} R\right) H_{m}^{(1)}\left(k_{0} R\right)} A_{m} .
\end{aligned}
$$

\section{APPENDIX E: PERTURBATION ORDERS OF THE 2D PROBLEM}

Any term in the Born series can be calculated in a recurrent manner with Eq. (8) using numerical integration, starting with 
$U_{0}(\mathbf{r})=U_{i}(\mathbf{r})$. The term of order $\ell+1$ can be calculated from term $\ell$ according to Eq. (E1).

$$
\begin{aligned}
U_{\ell+1}(\mathbf{r}) & =k_{0}^{2} \int_{\Omega} G\left(\mathbf{r}, \mathbf{r}^{\prime}\right) U_{\ell}\left(\mathbf{r}^{\prime}\right) \Delta \varepsilon_{r} d \mathbf{r} \\
& =\frac{i k_{0}^{2} \Delta \varepsilon_{r}}{4} \int_{0}^{R} \int_{0}^{2 \pi} H_{0}^{(1)}\left(k_{0}\left|\mathbf{r}-\mathbf{r}^{\prime}\right|\right) U_{\ell}\left(\mathbf{r}^{\prime}\right) r^{\prime} d \varphi^{\prime} d r^{\prime} .
\end{aligned}
$$

To proceed we employ the Hankel addition theorem

$$
H_{0}^{(1)}\left(k_{0}\left|\mathbf{r}-\mathbf{r}^{\prime}\right|\right)= \begin{cases}\sum_{n=-\infty}^{+\infty} J_{n}\left(k_{0} r\right) H_{n}^{(1)}\left(k_{0} r^{\prime}\right) \exp \left[\operatorname{in}\left(\varphi-\varphi^{\prime}\right)\right], & r \leqslant r^{\prime}, \\ \sum_{n=-\infty}^{+\infty} J_{n}\left(k_{0} r^{\prime}\right) H_{n}^{(1)}\left(k_{0} r\right) \exp \left[\operatorname{in}\left(\varphi-\varphi^{\prime}\right)\right], & r \geqslant r^{\prime},\end{cases}
$$

in which for $r$ we can distinguish between points inside and outside the cylinder.

Outside the cylinder. For all points $r$ outside the cylinder $r \geqslant r^{\prime}$ holds, such that applying the Hankel addition theorem results in one integral. See Eq. (E3a).

Inside the cylinder. Now both regimes of the Hankel addition theorem apply, such that the integral over $r$ is split into two integrals; one for 0 to $r$ and one for $r$ to $R$. See Eq. (E3b). We choose $n=m$ so that the same number of Bessel modes are used in the calculation.

$$
\begin{aligned}
U_{\ell+1}(r, \varphi)= & \frac{i k_{0}^{2} \Delta \varepsilon_{r}}{4} \sum_{n=-\infty}^{+\infty} \exp [i n \varphi] H_{n}^{(1)}\left(k_{0} r\right) \int_{0}^{R} \int_{0}^{2 \pi} J_{n}\left(k_{0} r^{\prime}\right) \exp \left[-i n \varphi^{\prime}\right] U_{\ell}\left(r^{\prime}, \varphi^{\prime}\right) r^{\prime} d \varphi^{\prime} d r^{\prime}, \\
U_{\ell+1}(r, \varphi)= & \frac{i k_{0}^{2} \Delta \varepsilon_{r}}{4} \sum_{n=-\infty}^{+\infty} \exp [i n \varphi]\left[H_{n}^{(1)}\left(k_{0} r\right) \int_{0}^{r} \int_{0}^{2 \pi} J_{n}\left(k_{0} r^{\prime}\right) \exp \left[-i n \varphi^{\prime}\right] U_{\ell}\left(r^{\prime}, \varphi^{\prime}\right) r^{\prime} d \varphi^{\prime} d r^{\prime}\right. \\
& \left.+J_{n}\left(k_{0} r\right) \int_{r}^{R} \int_{0}^{2 \pi} H_{n}^{(1)}\left(k_{0} r^{\prime}\right) \exp \left[-i n \varphi^{\prime}\right] U_{\ell}\left(r^{\prime}, \varphi^{\prime}\right) r^{\prime} d \varphi^{\prime} d r^{\prime}\right] .
\end{aligned}
$$

We can use the Fourier decomposition of $U_{\ell}(r, \varphi)$,

$$
U_{\ell}(r, \varphi)=\sum_{m=-\infty}^{+\infty} \hat{U}_{\ell}(r, m) \exp (i m \varphi),
$$

to calculate $U_{\ell+1}(r, \varphi)$ more conveniently. Substituting this in Eqs. (E3b) and (E3a), we get a double summation over $n$ and $m$ and the double integrals over $r^{\prime}$ and $\varphi^{\prime}$ become separable in both cases. The integral over $\varphi^{\prime}$ evaluates to

$$
\int_{0}^{2 \pi} \exp \left[-i n \varphi^{\prime}\right] \exp \left[i m \varphi^{\prime}\right] d \varphi^{\prime}=2 \pi \delta_{n m},
$$

with $\delta_{n m}$ a Kronecker delta, reducing the double summation to one over $m$ which is again of the same form as Eq. (E4). Therefore we find for $\hat{U}_{\ell}(r, m)$ the relations in Eqs. (E6a) and (E6b) for points outside and inside the cylinder respectively. We see that we can calculate all terms in the Born series by only computing integrals over the radial component in Fourier domain because of the orthogonality of the complex exponentials in this geometry. Given the expansion of $U_{0}(r, \varphi)$, which in our case is $\hat{U}_{0}(r, m)=$ $i^{m} J_{m}\left(k_{0} r\right)$, we can calculate $\hat{U}_{\ell}(r, m)$ up to any $\ell$, which then is transformed back to $U_{\ell}(r, \varphi)$ using Eq. (E4). This can all be performed numerically, but we do provide the analytical expressions for the first term $\hat{U}_{1}(r, m)$ for points inside and outside the cylinder in Eqs. (E7a) and (E7b), respectively, since the integrals in Eqs. (E6a) and (E6b) can be evaluated analytically for $\ell=0$ [33].

$$
\begin{aligned}
\hat{U}_{\ell+1}(r, m) & =\frac{i \pi k_{0}^{2} \Delta \varepsilon_{r}}{2} H_{m}^{(1)}\left(k_{0} r\right) \int_{0}^{R} J_{m}\left(k_{0} r^{\prime}\right) \hat{U}_{\ell}\left(r^{\prime}, m\right) r^{\prime} d r^{\prime}, \\
\hat{U}_{\ell+1}(r, m) & =\frac{i \pi k_{0}^{2} \Delta \varepsilon_{r}}{2}\left[H_{m}^{(1)}\left(k_{0} r\right) \int_{0}^{r} J_{m}\left(k_{0} r^{\prime}\right) \hat{U}_{\ell}\left(r^{\prime}, m\right) r^{\prime} d r^{\prime}+J_{m}\left(k_{0} r\right) \int_{r}^{R} H_{m}^{(1)}\left(k_{0} r^{\prime}\right) \hat{U}_{\ell}\left(r^{\prime}, m\right) r^{\prime} d r^{\prime}\right], \\
\hat{U}_{1}(r, m) & =\frac{i \pi k_{0}^{2} \Delta \varepsilon_{r}}{2} i^{m} \frac{R^{2}}{2}\left[J_{m}^{2}\left(k_{0} R\right)-J_{m-1}\left(k_{0} R\right) J_{m+1}\left(k_{0} R\right)\right) H_{m}^{(1)}\left(k_{0} r\right), \\
\hat{U}_{1}(r, m) & =\frac{i \pi k_{0}^{2} \Delta \varepsilon_{r}}{2} i^{m}\left\{\frac{-i r}{\pi k_{0}} J_{m}^{\prime}\left(k_{0} r\right)+\frac{R^{2}}{2} J_{m}\left(k_{0} r\right)\left[J_{m-1}\left(k_{0} R\right) H_{m}^{(1) \prime}\left(k_{0} R\right)-J_{m}\left(k_{0} R\right) H_{m-1}^{(1) \prime}\left(k_{0} R\right)\right]\right\} .
\end{aligned}
$$

[1] D. L. Colton and R. Kress, Inverse Acoustic and Electromagnetic Scattering Theory, 2nd ed. (Springer, Heidelberg, 1998).
[2] M. N. O. Sadiku, Numerical Techniques in Electromagnetics, 2nd ed. (CRC, Boca Raton, 2001). 
[3] A. Taflove and S. C. Hagness, Computational Electrodynamics: The Finite-Difference Time-Domain Method (Artech House, Boston, 1995).

[4] M. G. Moharam and T. K. Gaylord, Rigorous coupled-wave analysis of planar-grating diffraction, J. Opt. Soc. Am. 71, 811 (1981).

[5] L. Li and C. W. Haggans, Convergence of the coupled-wave method for metallic lamellar diffraction gratings, J. Opt. Soc. Am. A 10, 1184 (1993).

[6] S. Weinberg, Quasiparticles and Born series, Phys. Rev. 131, 440 (1963)

[7] M. Born and E. Wolf, Principle of optics, 7th ed. (Cambridge University Press, Cambridge, 2003).

[8] L. Novotny and B. Hecht, Principles of Nano-Optics, 1st ed. (Cambridge University Press, Cambridge, 2006).

[9] R. Bennett, Born-series approach to the calculation of Casimir forces, Phys. Rev. A 89, 062512 (2014).

[10] A. S. Bereza, A. V. Nemykin, S. V. Perminov, L. L. Frumin, and D. A. Shapiro, Light scattering by dielectric bodies in the Born approximation, Phys. Rev. A 95, 063839 (2017).

[11] C. M. Bender and S. A. Orszag, Advanced Mathematical Methods for Scientists and Engineers I: Asymptotic Methods and Perturbation Theory (Springer, New York, 1999).

[12] M. Znojil, Iterative solutions of the Lippmann-Schwinger-type equations, Phys. Rev. A 34, 2697 (1986).

[13] D. J. Kouri and A. Vijay, Inverse scattering theory: Renormalization of the Lippmann-Schwinger equation for acoustic scattering in one dimension, Phys. Rev. E 67, 046614 (2003).

[14] R. E. Kleinman, G. F. Roach, and P. M. van den Berg, Convergent Born series for large refractive indices, J. Opt. Soc. Am. A 7, 890 (1990).

[15] S. Tani, Padé approximant in potential scattering, Phys. Rev. 139, B1011 (1965).

[16] J. L. Gammel and F. A. McDonald, Application of the Padé approximant to scattering theory, Phys. Rev. 142, 1245 (1966).

[17] J. A. Tjon, Padé approximants in three-body calculations, Phys. Rev. D 1, 2109 (1970).

[18] W. M. Kloet and J. A. Tjon, Elastic neutron-deuteron scattering with local potentials, Ann. Phys. 79, 407 (1973).

[19] S. Roy, S. F. Pereira, H. P. Urbach, X. Wei, and O. El Gawhary, Exploiting evanescent-wave amplification for subwavelength low-contrast particle detection, Phys. Rev. A 96, 013814 (2017).

[20] O. El Gawhary, N. Kumar, S. F. Pereira, W. M. J. Coene, and H. P. Urbach, Performance analysis of coherent optical scatterometry, Appl. Phys. B 105, 775 (2011).

[21] S. Roy, O. El Gawhary, N. Kumar, S. F. Pereira, and H. P. Urbach, Scanning effects in coherent Fourier scatterometry, J. Eur. Opt. Soc. Rap. Public. 7, 12031 (2012).

[22] F. Simonetti, Multiple scattering: The key to unravel the subwavelength world from the far-field pattern of a scattered wave, Phys. Rev. E 73, 036619 (2006).

[23] F. Simonetti, L. Huang, N. Duric, and O. Rama, Imaging beyond the Born approximation: An experimental investigation with an ultrasonic ring array, Phys. Rev. E 76, 036601 (2007).

[24] F. C. Chen and W. C. Chew, Experimental verification of super resolution in nonlinear inverse scattering, Appl. Phys. Lett. 72, 3080 (1998).

[25] O. El Gawhary, M. C. Dheur, S. F. Pereira, and J. J. M. Braat, Extension of the classical Fabry-Perot formula to 1D multilayered structures, Appl. Phys. B 111, 637 (2013).

[26] O. J. F. Martin, A. Dereux, and C. Girard, Iterative scheme for computing exactly the total field propagating in dielectric structures of arbitrary shape, J. Opt. Soc. Am. A 11, 1073 (1994).

[27] P. B. Johnson and R. W. Christy, Optical constants of the noble metals, Phys. Rev. B 6, 4370 (1972).

[28] D. E. Aspnes and A. A. Studna, Dielectric functions and optical parameters of $\mathrm{Si}, \mathrm{Ge}, \mathrm{GaP}, \mathrm{GaAs}, \mathrm{GaSb}, \mathrm{InP}$, InAs, and $\mathrm{InSb}$ from 1.5 to $6.0 \mathrm{eV}$, Phys. Rev. B 27, 985 (1983).

[29] J. J. Bowman, T. B. A. Senior, P. L. E. Uslenghi, and J. S. Asvestas, Electromagnetic and Acoustic Scattering by Simple Shapes (Hemisphere, New York, 1987).

[30] R. F. Harrington, Time-Harmonic Electromagnetic Fields (IEEE, New York, 2001).

[31] M. Kerker, The Scattering of Light and Other Electromagnetic Radiation (Academic, New York, 2013).

[32] F. Frezza, F. Mangina, and N. Tedeschi, Introduction to electromagnetic scattering: Tutorial, J. Opt. Soc. Am. A 35, 163 (2018).

[33] Y. L. Luke, Integrals of Bessel Functions (McGraw-Hill, New York, 1962). 